\title{
A Case Report of Nail Patella Syndrome and Knee Pain
}

\section{Gupta Sunny ${ }^{1}$, Patel Mitesh ${ }^{1 *}$, Chhipa Irfan ${ }^{1}$, Barrientos Steven ${ }^{2}$ and Grzywinski Matthew ${ }^{3}$}

${ }^{1}$ Rothman Orthopaedic Institute, Sports Medicine, USA

${ }^{2}$ Department of Family Medicine, Rowan University School of Osteopathic

Medicine, New Jersey, USA

${ }^{3}$ Sidney Kimmel Medical College at Thomas Jefferson University, USA

*Corresponding Author: Patel Mitesh, Rothman Orthopaedic Institute, Sports

Medicine, USA.

DOI: $10.31080 /$ ASOR.2020.03.0201
Received: June 19, 2020

Published: July 30, 2020

(C) All rights are reserved by Patel Mitesh., et

al.

\begin{abstract}
Nail-Patella Syndrome (NPS), also known as Fong's Disease, Turner-Keiser Syndrome or Hereditary Onychoosteodysplasia (HOOD), is a rare hereditary condition affecting 1 in 50,000 individuals. The condition is associated with mutations in the LMX1B gene and is inherited in an autosomal dominant pattern. Patients with this syndrome may have several musculoskeletal abnormalities including hypoplastic or absent patellae, underdeveloped elbows, and iliac horns. The joint abnormalities associated with this condition predispose patients to osteoarthritis $(\mathrm{OA})$ and easy joint dislocation. Other common findings include nail abnormalities (absent or hypoplastic nails, nail ridges, and nail discoloration), renal dysfunction, glaucoma, irritable bowel syndrome, rash, and neuropathy. Here, we describe the case of a patient with known NPS presenting with bilateral knee pain.
\end{abstract}

Keywords: Nail-Patella Syndrome; Fong Disease; Turner-Keiser Syndrome; Iliac Horns

\section{Background}

Osteoarthritis is the most common cause of disability in older adults. Age, history of trauma, obesity, lack of exercise, and female gender increase an individual's risk of developing OA [1]. Some genetic conditions, such as NPS, also cause knee pain and predispose a patient to develop $\mathrm{OA}$ [2]. In this case report we present a case of knee OA in a patient with known NPS.

\section{Case Presentation}

The patient in this case is a 64-year-old male diagnosed with Nail-Patella Syndrome (NPS) in childhood based on incidental imaging findings. Iliac horns were discovered on previous X-rays during his childhood. He reports a history of normal knee function including playing sports as a child. He works in manufacturing and spends most of the workday on his feet. He has no history of knee surgery.
He presented with approximately 10 years of insidious onset bilateral knee pain. The pain is diffuse, worse with prolonged standing, and relieved by rest. He did not recall any trauma to the knees. Several years before presenting, he underwent chiropractic treatment with some relief. He denied knee clicking, locking, or instability.

On exam, the patient was $5^{\prime} 6^{\prime \prime}$ tall and weighed $137 \mathrm{lbs}$ with a BMI of 22. Inspection revealed no ecchymosis. Both patellae were palpable and smaller than expected. There was joint line tenderness with no effusion bilaterally. Range of motion was 0-100 degrees, strength was 5/5 in flexion and extension, and no instability to valgus and varus stress testing was noted. McMurray's test and lachman's exam was negative bilaterally.

Four view x-rays of bilateral knees demonstrated Kellgren Lawrence grade 2 degenerative changes in the medial and patellofemo- 
ral compartments, chondrocalcinosis, and hypoplastic patella (Figure 1).

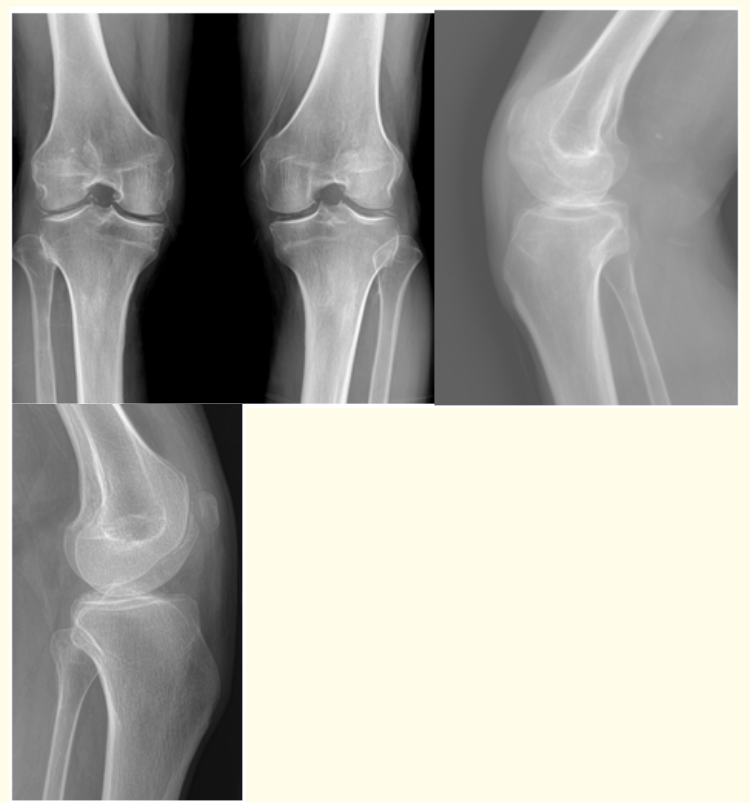

Figure 1: Multiple x-ray views of the patient's knees illustrating hypoplastic patellae and degenerative changes.

\section{Treatment}

After his initial visit, various conservative treatment options with discussed with the patient at length, including watchful waiting, activity modification, nutraceuticals, non-steroidal antiinflammatory medications, physical therapy, steroid injections, and viscosupplementation. The topic of possible surgery was also discussed if non-operative measures failed. He elected to proceed with a local injection of bupivacaine and kenalog in both knees to reduce inflammation and pain. The patient will also pursue formal physical therapy and return in four to six weeks for a follow up to his progress.

\section{Outcome and follow-up}

The patient followed up seven weeks later and reported the injections provided several days of relief. He was started on pregabalin by another provider for shoulder pain. He felt his knee pain had improved since starting this new medication. He was also offered viscosupplementation injections as a step up in treatment. If conservative measures fail this patient will be referred for reconstructive surgery. This would include patellofemoral ligament reconstruction, tibial tuberosity transposition, patellofemoral arthroplasty, or total knee arthroplasty [9].

\section{Discussion}

NPS was first described by Chatelain in 1820 [3]. Inheritance follows an autosomal dominant pattern and is associated with loss of function mutations in the LMX1B gene on chromosome 9 at 9q34.1 $[4,5]$. This gene is responsible for a family of transcription factors involved in ventral-dorsal body pattern formation during development [7]. Variable expressivity is responsible for the variation in features seen in different patients [2].

The diagnosis of NPS can be confirmed by genetic testing but is generally made clinically based on musculoskeletal findings [2]. Most patients are diagnosed in their 30 s, often by incidental findings on imaging. Iliac horns (Figure 2) are considered pathognomonic for NPS and are seen in $80 \%$ of patients [6]. The iliac 'horns' are bony outcrops located at the attachment site of the gluteus medius on the pelvis, which project posterolaterally [9]. These horns can be palpated on physical exam and are visible on third trimester fetal ultrasounds. They often are asymptomatic but can present as hip or back pain $[6,7]$. Besides iliac horns, clavicular horns have also been reported in patients with NPS [9].

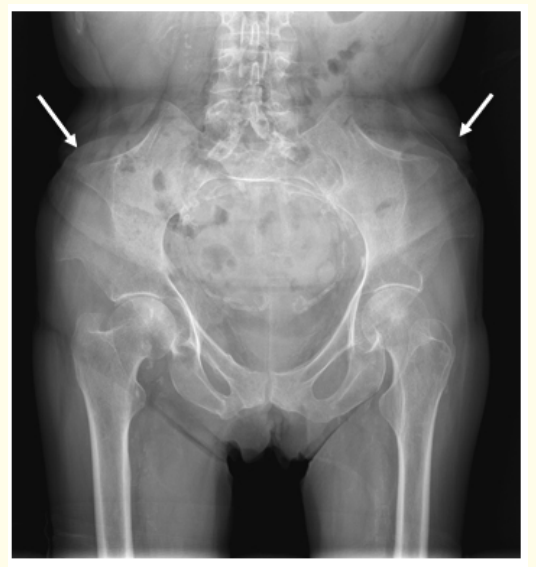

Figure 2: An example of the iliac horns seen in NPS that are pathognomonic [6].

Knee abnormalities, primarily patella aplasia and hypoplasia are seen in greater than $80 \%$ of patients [2]. The patella abnormalities can be unilateral or bilateral [2]. The abnormalities may be asymptomatic but $74 \%$ of NPS patients experience knee pain [8]. Osteoarthritis (OA) is common and may be accelerated in NPS [2]. $50 \%$ of patients experience some degree of patellar instability [8]. The high prevalence of knee issues cause NPS patients to seek medical attention. First line treatment for patients with NPS 
include conservative non-surgical approaches, for example physical therapy, bracing, NSAIDs, and steroid injections [9]. If patients remain symptomatic after conservative approaches, then a surgical evaluation may be required. The type of surgery varies depending on the patient's anatomy. Some procedures include tibial tubercle transfer, anteriorization of the tibial tubercle, total knee arthroplasty, and Krogius-Lecere procedure [4,9]. In NPS patients who underwent surgical intervention, $71 \%$ of patients reported they were satisfied with the outcome [8].

In addition to knee complaints, other joint problems may develop. Various elbow pathologies, such as cubitus valgus, hypoplastic radial heads, hypoplastic lateral condyles of the humeral head, flexion contracture, and antecubital pterygium, have all been reported $[2,10]$. Risk of elbow dislocation in patients with NPS is significant. One study found 27 of 44 patients had experienced radial head subluxation or dislocation [9].

Nail-Patella Syndrome not only can affect major joints, but it can also affect major organ systems. NPS patients tend to have abnormalities of the fingernails and toenails including hypoplastic nails, ridged nails, and discoloration. The thumb nail is the fingernail most commonly affected [2]. Renal dysfunction may occur in $30-60 \%$ of patients, with nephrotic syndrome and proteinuria as the most common findings $[2,6]$. Unfortunately, up to $20 \%$ of these patients experience renal failure and $10 \%$ required dialysis or renal transplant [6]. One case report noted effects during pregnancy, including worsening of proteinuria [11]. One-third of NPS individuals may experience irritable bowel syndrome and $10 \%$ of patients may have some degree of open angle glaucoma [6]. Neuropathy and abnormal skin findings, such as scaly erythematous plaques, have also been reported [2,5].

\section{Conclusion}

Nail-Patella Syndrome (NPS) is associated with various musculoskeletal abnormalities including hypoplastic or absent patellae, underdeveloped elbows, and iliac horns. These patients are at increased risk for joint dislocation and osteoarthritis (OA). Patients with NPS deserve a multi-disciplinary approach to their medical ailments, since this genetic condition may affect multiple organ systems. Many patients may be successfully treated with non-operative measures. But, if these measures do not relieve symptoms, then surgical consultation should be considered.

\section{Bibliography}

1. Wittenauer R., et al. "Background paper 6.12 osteoarthritis". World Health Organization (2013).
2. Nail-patella syndrome. National Institute of Health Genetic and Rare Disease Information Center (2018).

3. Konrad C., et al. "Nail-patella syndrome in a young patient followed up over 10 years: relevance of the sagittal trochlear septum for patellofemoral pathology". SICOT-J 2.26 (2016): 1-4.

4. Beguiristain JL., et al. "Nail-patella syndrome: long term evolution". Journal of Pediatric Orthopaedics 12.1 (2003): 13-16.

5. Mukai M., et al. "A familiar case of nail patella syndrome with heterozygous in-frame indel mutation in the LIM domain of LMX1B". Journal of Dermatology Science 90.1 (2018): 90-93.

6. Waziri TM., et al. "Hips don't lie: Fong Disease". Journal of the Belgian Society of Radiology 101.1 (2017): 1-2.

7. West JA and Louis TH. "Radiographic findings in the nail-patella syndrome". Baylor University Medical Center Proceedings 28.3 (2015): 334-336.

8. Tigchelaar S., et al. "Nail patella syndrome: knee symptoms and surgical outcomes". Orthopaedics and Traumatology: Surgery and Research 101.8 (2015): 959-962.

9. Louboutin L., et al. "Management of patellar problems in skeletally mature patients with nail-patella syndrome". Knee Surgery, Sports Traumatology, Arthroscopy 25.10 (2017): 30123016.

10. Kundu ZS and Siwach RC. "Nail-patella syndrome". Indian Journal of Medical Research 147.14 (2018): 619-620.

11. Aboobacker IN., et al. "Nail-Patella syndrome: A rare cause of nephrotic syndrome in pregnancy". Indian Journal of Nephrology 28.1 (2018): 76-78.

\section{Assets from publication with us}

- Prompt Acknowledgement after receiving the article

- Thorough Double blinded peer review

- Rapid Publication

- Issue of Publication Certificate

- High visibility of your Published work

Website: www.actascientific.com/

Submit Article: www.actascientific.com/submission.php

Email us: editor@actascientific.com

Contact us: +919182824667 